\title{
Winston-Lutz-Gao Test on the True Beam STx Linear Accelerator
}

\author{
Junfang Gao ${ }^{1 *}$, Xiaoqian Liu ${ }^{2}$ \\ ${ }^{1}$ Northeast Louisiana Cancer Institute, Monroe, LA, USA \\ ${ }^{2}$ Division of Pharmacy, The University of Texas MD Anderson Cancer Center, Houston, TX, USA \\ Email: *Junfang.gao@cancerinstitute.com
}

How to cite this paper: Gao, J.F. and Liu, X.Q. (2019) Winston-Lutz-Gao Test on the True Beam STx Linear Accelerator. International Journal of Medical Physics, Clinical Engineering and Radiation Oncology, 8, 9-20.

https://doi.org/10.4236/ijmpcero.2019.8100 2

Received: December 20, 2018

Accepted: January 18, 2019

Published: January 21, 2019

Copyright ( 2019 by author(s) and Scientific Research Publishing Inc. This work is licensed under the Creative Commons Attribution International License (CC BY 4.0).

http://creativecommons.org/licenses/by/4.0/

(c) (i) Open Access

\begin{abstract}
In the linear accelerator-based stereotactic radio surgery (SRS) and stereotactic body radiotherapy (SBRT) programs, single isocenter-multiple metastases' treatment has become more and more popular due to their high efficiency in treatment time. However, the absence of a comprehensive quality assurance program is still the challenge for medical physicists. The Winston-Lutz-Gao test, which we developed two years ago, was performed for the first time on a True Beam STx (Varian Medical System) linear accelerator in this study. Beams were designed by Eclipse with gantry, collimator, and couch full rotations, and a 200-pound weight was placed on the couch to mimic real treatment. The "frameless SRS QA target pointer" from the Brainlab company, with a $3.5-\mathrm{mm}$ metallic ball embedded in the center, was used as a phantom. Images were acquired by the portal imager built-in linear accelerator and analyzed directly by the Image browser in ARIA. We found that the farther the metastases were from the linac isocenter, the worse the congruence was between the beam mechanical and the radiation center. The farthest metastases should be within $6 \mathrm{~cm}$ from the linac isocenter per the AAPM TG-142 and American Society for Radiation Oncology (ASTRO) white paper criteria. To the best of our knowledge, this is the first off-isocenter Winston-Lutz test performed on a True Beam STx linear accelerator.
\end{abstract}

\section{Keywords}

Winston-Lutz-Gao Test, off-Iso Winston-Lutz Test, Single Iso-Multiple Mets' Treatment

\section{Introduction}

In recent decades, stereotactic radio surgery (SRS) and stereotactic body radio- 
therapy (SBRT) have been heavily implemented and researched in the radiation oncology field due to their unique medical and financial merits to cancer patients. Among SRS and SBRT practice the multiple cranial metastases treatment advanced faster than did any other kind of lesion treatment in both clinical and technique aspect. From Sahgal et al.'s recent comprehensive review in $\mathrm{Neo}$ ro-Oncology [1], the SRS alone has a superior patient survival rate compared with adjuvant whole brain radiation therapy (WBRT) for patients with up to four brain metastases (mets); even the adjuvant WBRT has a better distant control rate than does SRS alone. This will trigger more SRS treatment for multiple cranial mets in the future. Sahgal et al. reviewed some of the pros and cons for various type of modern SRS technologies, including the Gamma knife, cyber knife, and linear accelerator (linac). Each modality has a unique mechanical and geometrical character, but the clinical data do not support one modality's superiority over the others.

The linac based SRS/SBRT technique is more popular than the Gamma knife and cyber knife because almost every radiation oncology site is equipped with a linac. The modern digital controlled linac (such as the Varian True Beam STx Edge series) has a variety of beam energies and a very accurate mechanical control system. It makes the SRS/SBRT program less expensive and faster treatment than other modalities. However, there are two challenges ahead of us when we treat multiple targets by linac. As Sahgal et al. noted, one challenge is the small-field dosimetry, which requires accurately measured beam data to model the beam in the computer planning system. The other challenge is the complexity of the mechanical and geometrical quality assurance (QA). It is well known that the poor QA program comprises coverage for multiple targets and easy cause radionecrosis. Roper et al. [2] and Faught et al. [3] demonstrated that current standard linac QA tolerance (recommended byTG-142) is not appropriate for the single iso-multiple met treatment. However, single iso-multiple mets treatment planning systems such as Element and Eclipse are already on the market. A standard QA procedure really needs to be established for these systems to ensure the same accuracy as that is attained with single iso-single met treatment in the medical physics community.

This study tried to meet one of the needs of the above-mentioned QA procedures. We will illustrate one practical QA test, which is called the Winston-Lutz-Gao test [4], on the True Beam STx linac for single iso-multiplemets treatment. This test is testing the congruence accuracy between the field mechanical center and radiation center when the asymmetric beam is implemented to treat off-iso multiple mets. It is different from the standard Winston-Lutz test which only tests the congruence accuracy between field mechanical center and radiation center for symmetric beam treating the single met at isocenter. We developed Winston-Lutz-Gao test two years ago for single iso-multiple mets treatment with use of an old Varian 21EX machine. Since then, several relevant articles [5] [6] [7] were published that started to verify either the dosimetric or geometric accuracy of the single iso-multiple mets treatment technique. We will 
discuss these articles later in this article. The standard Winston-Lutz test was reviewed by Rowshanfarzad et al. [8] and carefully researched by $\mathrm{Du}$ et al. [9] [10]. Current tests are performed with full gantry, collimator, and couch three-axis rotation, and a 200-pound weight is used on the couch to mimic real clinical situations. Four locations, including one at the isocenter and three off from the isocenter (off-iso), have been investigated. We directly measured the linac robust specifications instead of its dosimetry delivery accuracy. Our results clearly indicated that the farther the locations from the isocenter, the worse the congruence between the field mechanical center and radiation center. We found that with our new True Beam STx with high-definition 120 multileaf collimator (HD 120 MLC) linac, the maximum off-iso distance limit from which to perform single iso-multiple mets treatment is $6 \mathrm{~cm}$. To make this procedure easier for use in the user clinical setting, we will elaborate the details of the test. The Eclipse plan is offered at no charge on request.

\section{Materials and Methods}

In the linac based SRS/SBRT program, the multileaf collimator (MLC) and Cone are two radiation beam collimators that conform the beam onto the planning target volume (PTV) or gross tumor volume (GTV). Therefore, the Winston-Lutz test should be performed on either the MLC or cone beam collimator, depending on the clinical need for each individual patient. During the single iso-multiple mets treatment, the MLC is the only option to date that can be used to conform the beam onto multiple targets. The Winston-Lutz-Gao test is performed only on the MLC in the Varian product environment in our clinic. A field size of $1 \mathrm{~cm} \times 1 \mathrm{~cm}$ square is created at four different locations where the off-isodistance from the isocenter (d) is $0,3,6,9 \mathrm{~cm}$ (Figure 1). The field shown in Figure 1 labeled " $\mathrm{d}=0 \mathrm{~cm}$ " is a symmetric field, and the remaining three fields are asymmetric fields. All of these squares were generated in the Eclipse V.13.7 by either a $2.5-\mathrm{mm}$ micro-MLC or a $5-\mathrm{mm}$ MLC. The red mark in the figure indicates the ISO position. There are several ways to create a $1 \mathrm{~cm} \times 1 \mathrm{~cm}$ micro-MLC or MLC square, such as creating a block and then copying the MLC to the block, or using the MLC Shape tool, or using Shaper directly. However, the easiest and most practical way to create each square is to type the MLC position in the beam MLC property after resetting all MLC positions; an example case is shown in Figure 2. We listed in Table 1 those MLC leaf pairs that needed to be adjusted to generate a corresponding square in Table 1 (only for a HD 120 MLC system such as the Varian True Beam STx, Edge and Novalis).

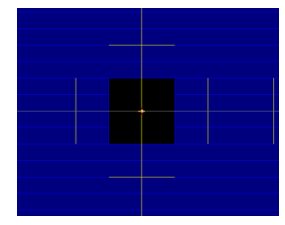

$\mathrm{d}=0 \mathrm{~cm}$

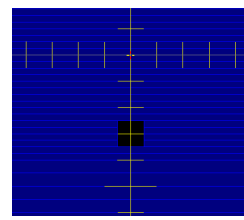

$\mathrm{d}=3 \mathrm{~cm}$

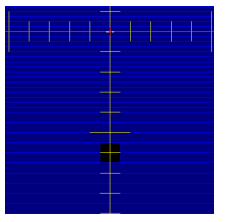

$\mathrm{d}=6 \mathrm{~cm}$

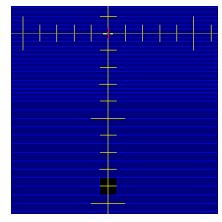

$\mathrm{d}=9 \mathrm{~cm}$

Figure 1. Off-iso distance between beam mechanical center and linac isocenter. 


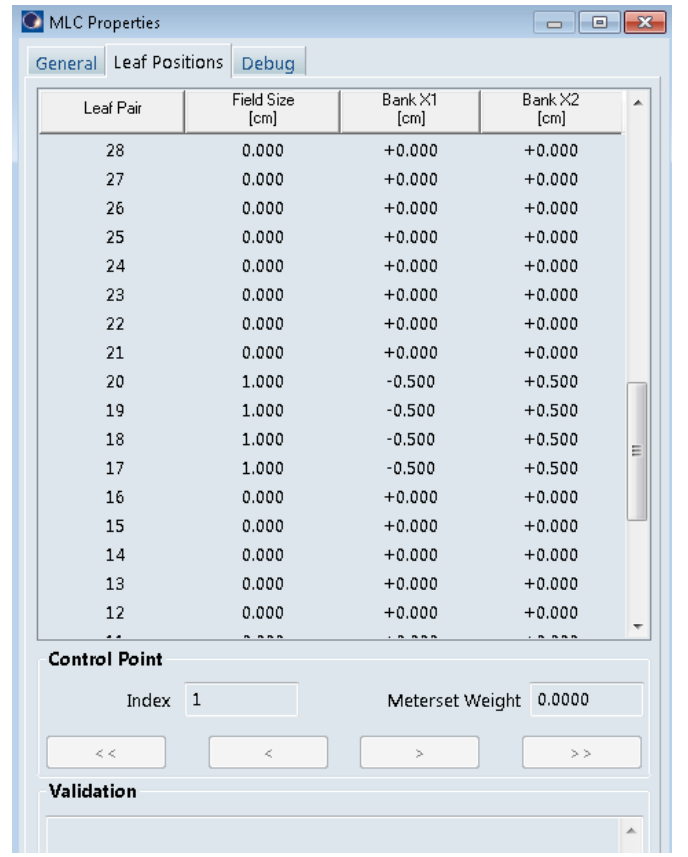

Figure 2. Adjusting the multileaf collimator (MLC) position in the MLC properties tab.

Table 1. Beam center locations and corresponding leaf pairs needed to be adjusted.

\begin{tabular}{ccccc}
\hline Off-iso distance & $\mathrm{d}=0 \mathrm{~cm}$ & $\mathrm{~d}=3 \mathrm{~cm}$ & $\mathrm{~d}=6 \mathrm{~cm}$ & $\mathrm{~d}=9 \mathrm{~cm}$ \\
\hline Leaf pairs that needed to be adjusted & $29,30,31,32$ & $17,18,19,20$ & 10,11 & 4,5 \\
\hline
\end{tabular}

Beam selection requires good experience with image acquisition and knowledge of collision zone of the system. For the standard Winston-Lutz test, Du et al. [9] made an extensive study of how to minimize the systematic and random errors of isocenter localization by proper selection of the gantry and collimator angles. They concluded that using opposing gantry four cardinal angles and opposing collimator angles can minimize the ISO center uncertainty to a large extent. In our tests, we also considered the couch rotation in our beam selection. Based on our experience, we believe that the uncertainty from the couch rotational axis has more influence than does the uncertainty from the gantry and collimator rotational axis. In contrast, in the hyper arc module in the True Beam STx2.7 version, the gantry, collimator, and couch might move at the same time during treatment, and the uncertainties of their three axes should be considered. During our test, we used two different beam schema for symmetric $(\mathrm{d}=0 \mathrm{~cm})$ and asymmetric $(\mathrm{d}=3,6,9 \mathrm{~cm})$ fields (Table 2 and Table 3$)$. For a symmetric field, we selected four opposing cardinal gantry angles and four opposing collimator angles (except for beam 1, the maximum collimator angle was $160^{\circ}$ ). For an asymmetric field, the collimator angle must be synchronized with the couch angle to acquire a portal image by an electronic portal imaging device (EPID). This will reduce options to choosing gantry, collimator, and couch angles to avoid gantry and couch collision. This is one of the complexities of the single iso-multiple mets treatment $\mathrm{QA}$ process. 
Table 2. Beam selection of eight beams for symmetric field $(\mathrm{d}=0 \mathrm{~cm})($ IEC 61217 scale).

\begin{tabular}{ccccccccc}
\hline Beam No. & 1 & 2 & 3 & 4 & 5 & 6 & 7 & 8 \\
\hline Gantry & 180 & 90 & 0 & 270 & 0 & 0 & 0 & 0 \\
Collimator & 160 & 90 & 0 & 270 & 90 & 45 & 315 & 270 \\
Couch & 0 & 0 & 0 & 0 & 90 & 45 & 315 & 270 \\
\hline
\end{tabular}

Table 3. Beam selection of eight beams for asymmetric field $(\mathrm{d}=3,6,9 \mathrm{~cm})($ IEC 61217 scale).

\begin{tabular}{ccccccccc}
\hline Beam No. & 1 & 2 & 3 & 4 & 5 & 6 & 7 & 8 \\
\hline Gantry & 180 & 90 & 0 & 270 & 0 & 0 & 0 & 0 \\
Collimator & 0 & 0 & 0 & 0 & 90 & 45 & 315 & 270 \\
Couch & 0 & 0 & 0 & 0 & 90 & 45 & 315 & 270 \\
\hline
\end{tabular}

In our tests, we considered the influence from couch rotational uncertainty more than other researchers did. To mimic real treatment situations, we loaded a 200-pound (about 90 kilogram) weight onto the couch (The used electron cerrobend block are used as weight here), which is the approximate average patient's weight in the United States. The weight distribution is similar to that of the normal patient body lying on the couch. Then the Wonston-Lutz-Gao test measure the congruence between the mechanical field and radiation field center as real patient was placed on table during treatment (Figure 3). This will include all potential factors that affect the congruence accuracy between the beam mechanical field and radiation field. The True Beam STx couches have six degrees of freedom. To avoid automatic correction of the image, we locked the couches in vertical, longitudinal, and lateral displacement and turned off the couch pitch and roll (keeping them at 0.0 always) during beam delivery. Once the phantom was aligned with crosshairs, only couch rotation was allowed.

The phantom we used in the test, manufactured by the Brainlab Company, was called "frameless SRS QA target pointer" (Figure 4). A metallic ball with a diameter of $3.5 \mathrm{~mm}$ is embedded into an acrylic block. The ball is accurately indicated by the block cross line on four sides for setup purposes. The block is connected with one rod and an adjusting device. Then the whole adjusting device is supported by a block base. The mechanical field of the linac is an open space projected by the mechanical components like jaws, or the MLC or cone beam collimator. It is represented by the light field. The center of the mechanical field can be found by using the mechanical distance indicator (MDI). After careful annual and monthly QA, the mechanical field center can be represented with high accuracy by the intersection of crosshairs in the light field at four cardinal gantry angles. In this test, the phantom with the metallic ball was aligned by the light field crosshairs (Same results as lasers if the lasers are carefully calibrated).Therefore, the metallic ball was aligned into the mechanical isocenter by the light field crosshairs. 


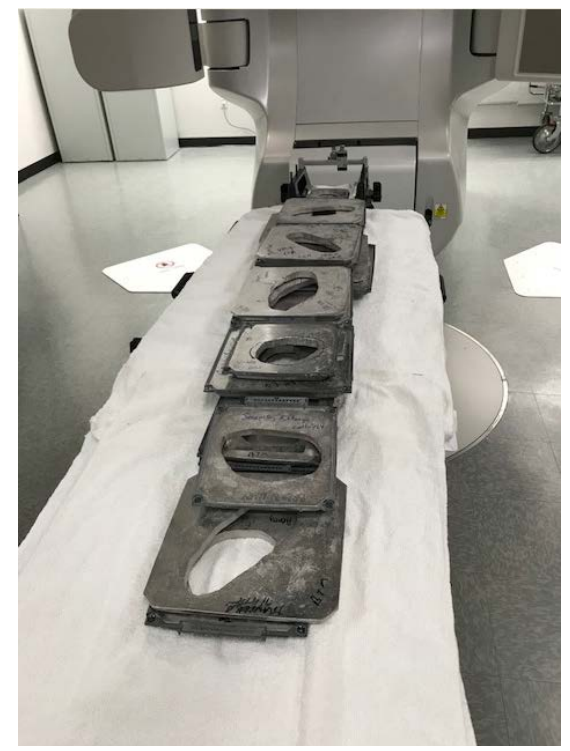

Figure 3. Weights (used cerrobend block) weighing 200 pounds were placed on the couch to imitate the patient weight.

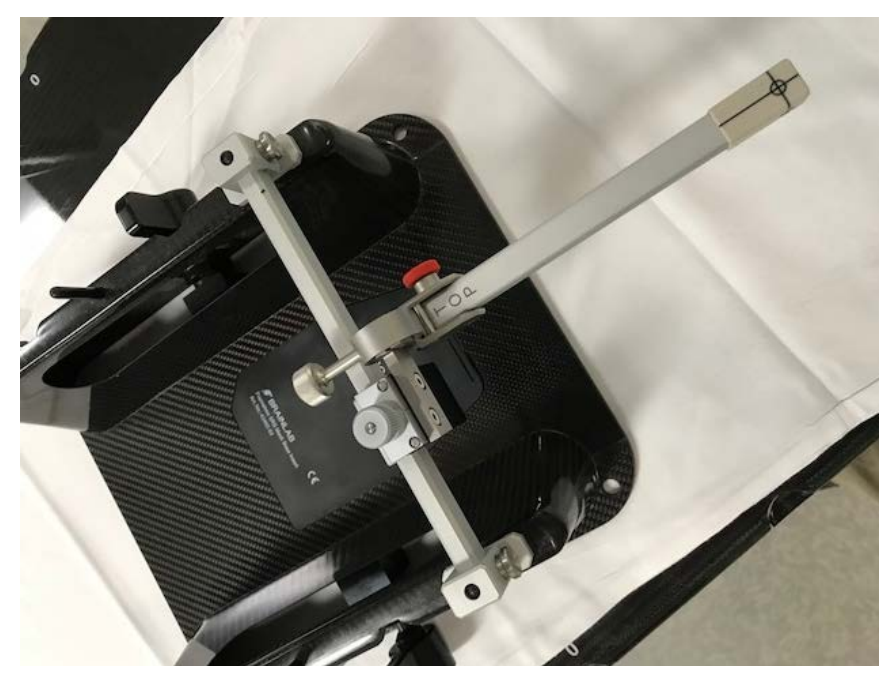

Figure 4. The phantom, adjusting device, and supporting base.

Imaging acquisition and analysis is the critical step in our test. The digital images were acquired by EPID, which is currently the most popular way to perform the Winston-Lutz test [8]. The portal image system was calibrated in our routine monthly QA program per the recommendation by TG-142. The image analysis is a broad pool in the medical physics community. Some institutions developed their in-house software to calculate the offset, whereas others use commercial software (such as ImagePro and PipsPro). Some venders and institutions use direct measurement offset from the images. Our experience shows that their accuracy is pretty much the same at the sub-millimeter level. We have commercial PipsPro software, but it can analyze only the symmetric image. Its accuracy also depends on the preset "Search Offset pixel number". We believe that the direct measurement method in the Image monitor in the treatment 
console or in the Image browser from ARIA (an electronic medical record system from Varian Company) is the most efficient and clinically acceptable method, with fewer numerical errors, even with some human error included. We validated the direct measurement methods by PipsPro for symmetric fields. They predicted offsets that were very close to each other. Therefore, the direct measurement method was used throughout the whole image analysis. Changing the gray level of image will change the shape of image but it will not change final results. This can be very easy verified.

\section{Results}

In this study, four locations were investigated, and eight beams were used at each location. In total, 32 images were acquired in the new True Beams STx linac. All images are offered at no charge on request if some readers are interested in using other tools to do the image analysis. To validate the direct measurement method, we compared the data acquired from PipsPro with data from direct measurement in Table 4. Although there were some differences between them, the average offset was exactly the same; the maximum offset was close and is acceptable in the practical clinic. To illustrate the direct measurement method in detail, one sample image is presented as in Figure 5, where four measurements were performed in the $\mathrm{u}$ and $\mathrm{v}$ coordinates axis. One sample calculation was also demonstrated as follows: The offset in the vertical direction is $\Delta \mathrm{u}=(0.33-$ $0.24) / 2=0.045 \mathrm{~cm}=0.45 \mathrm{~mm}$, and in horizontal direction is $\Delta \mathrm{v}=(0.32-$ $0.25) / 2=0.035 \mathrm{~cm}=0.35 \mathrm{~mm}$. The total offset is $R=\sqrt{(\Delta u)^{2}+(\Delta v)^{2}}=0.57$ $\mathrm{mm}$. The image in Figure 5 was acquired in the filter "Optimized" in the Image browser in ARIA. The window level affects the appearance of the image but does not substantially affect the offset measurement accuracy of the calculation. All measurement data were carefully collected from images (Table 5). The maxium and average offset vs off-iso distance is plotted in Figure 6. In this figure, when

Table 4. Offset data acquired from direct measurement and PipsPro (unit is $\mathrm{mm}$ ).

\begin{tabular}{ccccccccccc}
\hline Beam No. & 1 & 2 & 3 & 4 & 5 & 6 & 7 & 8 & Avg. & Max \\
\hline Direct & 0.11 & 0.15 & 0.35 & 0.16 & 0.52 & 0.35 & 0.38 & 0.52 & 0.32 & 0.52 \\
PipsPro & 0.12 & 0.23 & 0.40 & 0.14 & 0.46 & 0.38 & 0.41 & 0.42 & 0.32 & 0.46 \\
\hline
\end{tabular}

Table 5. The offset between beams' mechanical center and radiation center was tableted. When the off-iso distance reached $6 \mathrm{~cm}$, the average offset was $0.77 \mathrm{~mm}$ and the maximum offset was $1.02 \mathrm{~mm}$.

\begin{tabular}{ccccccccccc}
\hline & \multicolumn{10}{c}{ Beam number } \\
\hline \multirow{2}{*}{$\mathrm{d}(\mathrm{mm})$} & 1 & 2 & 3 & 4 & 5 & 6 & 7 & 8 & \multirow{8}{*}{ Max offset } & Avg. offset \\
\cline { 2 - 9 } & \multicolumn{10}{c}{ Offset $(R)$} \\
\hline 0 & 0.11 & 0.15 & 0.35 & 0.16 & 0.52 & 0.35 & 0.38 & 0.52 & 0.52 & 0.32 \\
3 & 0.25 & 0.46 & 0.52 & 0.30 & 0.80 & 0.60 & 0.66 & 0.76 & 0.80 & 0.54 \\
6 & 0.32 & 0.57 & 0.81 & 0.60 & 1.02 & 1.01 & 0.85 & 0.95 & 1.02 & 0.77 \\
9 & 1.00 & 0.74 & 0.63 & 0.65 & 1.11 & 1.15 & 0.95 & 1.05 & 1.15 & 0.91 \\
\hline
\end{tabular}




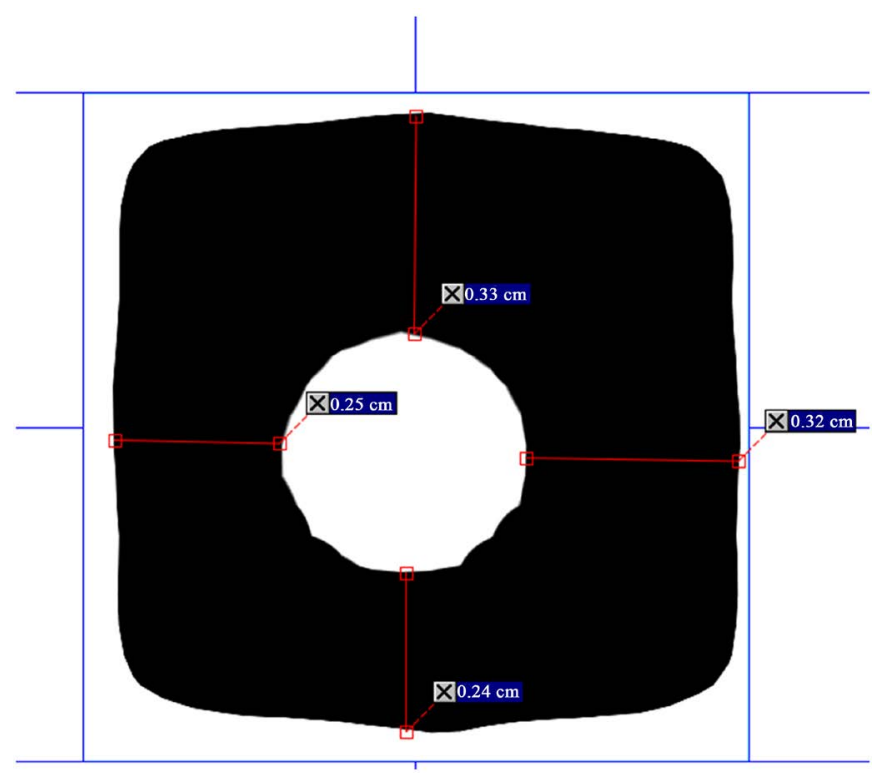

Figure 5. One sample image acquired from the Electronic Portal Imaging Device (EPID).

\section{Offset vs. Off-iso distance}

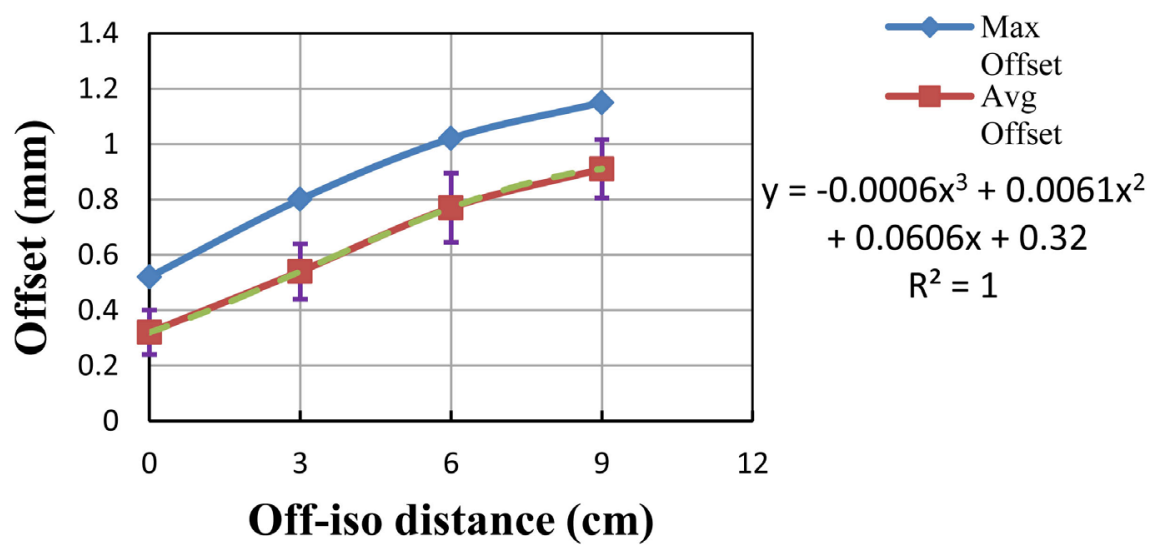

Figure 6. Maximum and average offset vs. off-iso distance. The first standard deviation is presented with average offset. The green dashed line is the cubic polynomial fit line. The cubic function is presented on the right.

the off-iso distance reached $6 \mathrm{~cm}$, the average offset was $0.77 \mathrm{~mm}$ and the maximum offset was $1.02 \mathrm{~mm}$. Due to the limitation of machine availability, the measurements are only performed at the off-iso distance of $3 \mathrm{~cm}, 6 \mathrm{~cm}$ and $9 \mathrm{~cm}$. The more detailed measurement in small pieces distance will be more time consuming.

The ASTRO whitepaper [11] requires that the "Winston-Lutz type of test provides the fundamental assessment of radiation isocenter and should be performed daily" before the SRS/SBRT procedure on the day of the procedure. The offset during the SRS/SBRT procedure is assumed to remain the same as that assessed by the Winston-Lutz test. Based on the ASTRO whitepaper [11] (Table 5), the "Winston-Lutz test-both cones and MLC, covering complete range of 
gantry, couch, collimator positions should be $\leq 0.75 \mathrm{~mm}$ average and $\leq 1 \mathrm{~mm}$ maximum". Per TG-142 II.C.3, based on the offset value, three action levels should be taken: Inspection Action, Scheduled Action, and Immediate Action. Also according to TG-142 [12], a single result that exceeds the tolerance value, but not excessively, should cause investigation or scheduled maintenance. Under these conditions, deviations may slightly exceed the tolerance, but the clinical impact over the course of a few days may not be significant." We adopted these recommendations as our clinical QA guideline. We also used the off-iso distance of $6 \mathrm{~cm}$ as our maximum limit for performing the single iso-mutiple mets treatment procedure. This criterion is widely adopted in our routine clinic in single iso-four mets or five mets patient treatment. It is also consistent with the University of Alabama at Birmingham (UAB) criteria.

\section{Discussion}

In the medical physics field, there appears to be some confusion between the Winston-Lutz test and the End-to-End test in the single iso-multiple mets treatment QA process. Some authors [2] [5] [6] tried to use the End-to-End test to replace Winston-Lutz test and to justify SRS/SBRT QA process. We think of the Winston-Lutz test as the linac performance test and the End-to-End test as the linac mission test. They are different and cannot replace each other. Performance tests focus machine mechanical and electrical specifications against industrial benchmarks and standards. It should be directly measured by calibrated tools and equipment, such as routine daily, monthly, annually quality assurance and the Winston-Lutz-Gao test in this study. Mission tests focus on actual usage to determine whether the machine can accomplish certain missions with certain percentage uncertainty, such as patient specific dosimetry QA and Houston IROC phantom irradiation. Mission tests depend not only on machine performance but also on beam modeling, the planning algorithm, phantom setup, beam delivery, and dosimetry analysis. The uncertainty from each component can either accumulate or cancel each other during the mission test. Mission test is a thorough test of the work flow accuracy while the performance test is a robust test of machine specification. The goal of radiation therapy is to deliver a dose to the patient, but the two types of test cannot override each other. The integration of both the machine performance test and the mission test into the institutional QA program is the only way to ensure high-quality treatment. Moliner et al. [13] demonstrated that even the linac collimator has $2^{\circ}-3^{\circ}$ mechanical error and MLC has $0.5 \mathrm{~mm}$ mechanical error, the patient specific QA still cannot $100 \%$ to detect the those errors.

During the Winston-Lutz-Gao test, the treatment planning system is used only for creating the $1 \mathrm{~cm} \times 1 \mathrm{~cm}$ square, and no dosimetry calculation is involved. Beam delivery and image acquisition are part of the standard Varian True Beam STx operation procedure. The distance measurement tool is very easy calibrated in monthly image calibration. Overall, the current test is a direct measurement against the Varian industrial standards. It is very easy to repeat, verify, and 
adopt by any clinic that is equipped with a True Beam STx linac. It is designed for practical clinical use. Figure 6 clearly shows that the farther the location from the linac isocenter, the larger is the deviation between the field mechanical center and radiation center. We discussed the cause in detail in our previous article [4]. This phenomenon is mainly attributed to mechanical and radiation uncertainty.

Another confusion in the medical physics field is on the understanding of tolerance limit of the Winston-Lutz test in the SRS/SBRT procedure. As stated in the "RESULTS" section, the ASTRO whitepaper [11] (Table 5) clearly stated that uncertainty limit should be $\leq 0.75 \mathrm{~mm}$ on average and $\leq 1 \mathrm{~mm}$ maximum. In 2016, we published our study of the first off-isocenter Winston-Lutz test on the single iso-multiple mets treatment [4]. In 20017, Calvo-Ortega et al. performed a similar study [6] with use of their in-house phantom. Calvo-Ortega et al. claimed that the maximum off-iso distance of the peripheral target is $6 \mathrm{~cm}$ on one 2100 $\mathrm{CD}$ Varian linac. Their conclusion is different from our finding, that $3 \mathrm{~cm}$ is the maximum off-iso distance on one 21EX Varian linac. After carefully reviewing their article, we believe there are three reasons for this difference. The first is their misunderstanding the tolerance limit. From Table 3 in their study, both the "Right" and "Left" target failed in the " $\leq 0.75 \mathrm{~mm}$ average and $\leq 1 \mathrm{~mm}$ maximum" criterion because two maximum limits are above $1 \mathrm{~mm}$ in the "Right" target test, and one maximum limit is above $1 \mathrm{~mm}$ in the "Left" target test. The maximum limit is $1.6 \mathrm{~mm}$ in one of the tests. The mean offset in both target tests was $0.8 \mathrm{~mm}$, which did not meet the " $\leq 0.75 \mathrm{~mm}$ average" criterion. The second reason is that they developed the phantom and dosimetry analysis in the mission test format. Five sources of uncertainty are included in their target delineation. All of these uncertainties either add up or cancel each other. Gamma analysis based on a $10 \% / 1 \mathrm{~mm}$ criterion should not be used to assess the measurement which requires $0.75-\mathrm{mm}$ accuracy. Automatic registration is also dependent on the filter and region of interest (ROI). The last reason might be caused by two different machines since each machine has its own unique mechanical and electric specifications and there is no identical machine in the world, even though some vendors claim they can do beam matching.

\section{Conclusion}

We performed a Winston-Lutz-Gao test on our new True Beam STx for single iso-multiple mets treatment. The test was developed with gantry, collimator, and couch in full-range rotation. To mimic patient treatment, a 200-pound (90-kilogram) weight was distributed on the couch. During this test, we clearly observed that the farther the location from the isocenter, the worse was the congruence between the beam mechanical and radiation center. We concluded that the off-iso distance for the farthest mets should be less than $6 \mathrm{~cm}$, per the TG-142 and the ASTRO white paper recommendation on the True Beam STx linac with $120 \mathrm{HD}$ MLC. To our knowledge, this is the first off-isocenter Winston-Lutz test performed on the True Beam STx linac. It will directly impact the 
accuracy of the True Beam-based single iso-multiple mets treatment. This is a practical clinic test, and all Eclipse plan and images are available at no charge on request. It is highly recommended that this test be included in institutions performing the single iso-multiple mets treatment procedure.

\section{Acknowledgements}

We thank Tamara K. Locke and the Department of Scientific Publications at the University of Texas MD Anderson Cancer Center for editorial review of the manuscripts.

\section{Conflicts of Interest}

The authors declare no conflicts of interest regarding the publication of this paper.

\section{References}

[1] Sahgal, A., Ruschin, M., Ma, L.J., Verbakel, W., Larson, D. and Brown, P.D. (2017) Stereotactic Radiosurgey Alone for Multiple Brain Metastases? A Review of Clinical and Technical Issues. Neuro-Oncology, 19, 2-15.

https://doi.org/10.1093/neuonc/nox001

[2] Roper, J., Chanyavanich, V., Betzel, G., Switchenko, J. and Dhabaan, A. (2015) Single-Isocenter Multiple-Target SRS: Risk of Compromised Coverage. International Journal of Radiation Oncology, Biology, Physics, 93, 540-546. https://doi.org/10.1016/j.ijrobp.2015.07.2262

[3] Faught, A.M., Trager, M., Yin, F.F., Kirkpatrick, J. and Adamson, J. (2016) Re-Examining TG-142 Recommendation in Light of Modern Techniques for Linear Accelerator Based Radiasurgery. Medical Physics, 43, 5437. https://doi.org/10.1118/1.4962471

[4] Gao, J. and Liu, X. (2016) Off-Isocenter Winston-Lutz Test for Stereotactic Radiosurgery/Stereotactic Body Radiotherapy. International Journal of Medical Physics, Clinical Engineering and Radiation Oncology, 5, 154-161. https://doi.org/10.4236/ijmpcero.2016.52017

[5] Poder, J., Brown, R., Porter, H., Gupta, R. and Ralston, A. (2018) Development of a Dedicated Phantom for Multi-Target Single-Isocenter Stereotactic Radiosurgery End to End Testing. Journal of Applied Clinical Medical Physics, 16, 1-10.

[6] Calvo-Ortega, J.F., Pozo, M., Moragues, S. and Casals, J. (2017) Targeting Accuracy of Single-Isocenter Intensity-Modulated Radiosurgery for Multiple Lesions. Medical Dosimetry, 42, 104-110. https://doi.org/10.1016/j.meddos.2017.01.006

[7] Tsuneda, M., Nishio, T., Saito, A., Tanaka, S., Suzuki, T., Kawahara, D., Matsushita, K., Nishio, A., Ozawa, S., Karasawa, K. and Nagata, Y. (2018) A Novel Verification Method Using a Plastic Scintillator Imagining System for Assessment of Gantry Sag in Radiotherapy. Medical Physics, 45, 2411-2423. https://doi.org/10.1002/mp.12922

[8] Rowshanfarzad, P., Sabet, M., O’Connor, D.J. and Greer, P.B. (2011) Isocenter Verification for Linac-Based Stereotactic Radiation Therapy: Review of Principles and Techniques. Journal of Applied Clinical Medical Physics, 12, 185-195. https://doi.org/10.1120/jacmp.v12i4.3645

[9] Du, W., Johnson, J.L., Jiang, W. and Kudchadker, R. (2016) On the Selection of Gantry and Collimator Angles for Isocenter Localization Using Winston-Lutz Tests. 
Journal of Applied Clinical Medical Physics, 17, 167-178.

https://doi.org/10.1120/jacmp.v17i1.5792

[10] Du, W., Gao, S., Jiang, W. and Kudchadker, R. (2018) Independent Evaluation of the Effectiveness of IsoCal in Improving Image Center Accuracy on Varian TrueBeam and Clinic Machines. Journal of Applied Clinical Medical Physics, 19, 1-8. https://doi.org/10.1002/acm2.12402

[11] Solberg, T., Balter, J.M., Benedict, S.H., Fraass, B., Kavanagh, B., Miyamoto, C., Pawlicki, T., Potters, L. and Yamada, Y. (2011) Quality and Safety Considerations in Stereotactic Radiosurgery and Stereotactic Body Radiation Therapy. Practical Radiation Oncology, 2, 2-9.

[12] Klein, E.E., Hanley, J., Bayouth, J., Yin, F.F., Simon, W., Dresser, S., Serago, C., Aguirre, F., Ma, L.J., Arjomandy, B., Liu, C., Sandin, C. and Holmes, T. (2009) Task Group 142 Report: Quality Assurance of Medical Accelerators. Medical Physics, 36, 4197-4212. https://doi.org/10.1118/1.3190392

[13] Moliner, G., Sorro, L., Verstraet, R., Daviau, P.A., Casas, M., Piron, B., Dubois, K., Debrigode, C., Barrau, C., Bons, F. and Greefier, J. (2018) Assessment of Combined Use of ArcCheck ${ }^{\circledast}$ Detector and Portal Dosimetry for Delivery Quality Assurance of Head and Neck and Prostate Volumetric-Modulated Arc Therapy. Journal of Applied Clinical Medical Physics, 19, 1-7. https://doi.org/10.1002/acm2.12460 